\title{
ASYMPTOTIC BEHAVIOR OF ALMOST-ORBITS OF NONEXPANSIVE SEMIGROUPS WITHOUT CONVEXITY
}

\author{
By Hirobumi KiUchi and Wataru Takahashi
}

\begin{abstract}
We first prove a resul on the asymptotic behavior of almost-orbits of nonexpansive semigroups without convexity in a Hilbert space. This is a generalization of results of Rodé [7] and Takahashi [10]. Further we prove a fixed point theorem for Lipschitzian semigroups without convexity. This is a generalization of results of Lau [3], Takahashi [8], [10] and Ishihara [2].
\end{abstract}

1. Introduction. Let $H$ be a real Hilbert space with norm $\|\cdot\|$ and inner product $(\cdot, \cdot)$ and let $C$ be a nonempty subset of $H$. A mapping $T: C \rightarrow C$ is said to be Lipschitzian if there exists a nonnegative number $k$ such that

$$
\|T x-T y\| \leqq k\|x-y\| \quad \text { for every } \quad x, y \in C
$$

and nonexpansive in the case of $k=1$. Let $S$ be a semitopological semigroup, i. e., a semigroup with a Hausdorff topology such that for each $s \in S$, the mappings $t \rightarrow t \cdot s$ and $t \rightarrow s \cdot t$ of $S$ into itself are continuous. Then a family $\mathcal{S}=$ $\left\{T_{s}: s \in S\right\}$ of mappings of $C$ into itself is called a Lipschitzian semigroup on $C$ if it satisfies the following:

(1) $T_{s t} x=T_{s} T_{t} x$ for all $s, t \in S$ and $x \in C$;

(2) for each $x \in C$, the mapping $s \rightarrow T_{s} x$ is continuous on $S$;

(3) for each $s \in S, T_{s}$ is a Lipschitzian mapping of $C$ into itself with Lipschitz constant $k_{s}$. A Lipschitzian semigroup $\mathcal{S}=\left\{T_{t}: t \in S\right\}$ on $C$ is said to be nonexpansive if $k_{s}=1$ for every $s \in S$. Recently, Takahashi [10] proved a nonlinear ergodic theorem and a fixed point theorem for nonexpansive semigroups without convexity in a Hilbert space. On the other hand, MiyaderaKobayasi [4] introduced the notion of an almost-orbit of nonexpansive semigroups and established the weak and strong almost convergence of such an almost-orbit; see also [1], [11], [12].

1980 Mathematics Subject Classification (1985 Revision). Primary 47A35, 47H09.

Key words and phrases. Fixed point, nonexpansive mapping, invariant mean, almostorbits.

Received July 24, 1991. 
In this paper, we first prove a result on the asymptotic behavior of almostorbits of nonexpansive semigroups without convexity in a Hilbert space. This is a generalization of results of Rodé [7] and Takahashi [10]. Further we prove a fixed point theorem for Lipschitzian semigroups without convexity. This is a generalization of results of Lau [3], Takahashi [8], [10] and Ishihara [2].

2. Asymptotic behavior of almost-orbits. Let $B(S)$ be the Banach space of all bounded real-valued functions on $S$ with supremum norm and let $X$ be a subspace of $B(S)$ containing constants. Then an element $\mu$ of $X^{*}$ (the dual of $X$ ) is a mean on $X$ if and only if

$$
\inf _{s \in S} f(s) \leqq \mu(f) \leqq \sup _{s \in S} f(s) \quad \text { for all } \quad f \in X .
$$

Let $\mu$ be a mean on $X$ and $f \in X$. Then, according to time and circumstances, we use $\mu_{t}(f(t))$ instead of $\mu(f)$. For each $s \in S$ and $f \in B(S)$, we define elements $l_{s} f$ and $r_{s} f$ in $B(S)$ given by

$$
\left(l_{s} f\right)(t)=f(s t) \text { and }\left(r_{s} f\right)(t)=f(t s) \quad \text { for all } t \in S .
$$

Let $X$ be a subspace of $B(S)$ containing constants and invariant under $l_{s}, s \in S$ $\left(r_{s}, s \in S\right)$. Then a mean $\mu$ on $X$ is said to be left invariant (right invariant) if

$$
\mu(f)=\mu\left(l_{s} f\right)\left(\mu(f)=\mu\left(r_{s} f\right)\right) \quad \text { for all } f \in X \text { and } s \in S .
$$

An invariant mean is a left and right invariant mean. We know following [9]: If $X$ is a left invariant subspace of $B(S)$ containing constants and $\mu$ is a left invariant mean on $X$, then for any $f \in X$,

$$
\sup _{s} \inf _{t} f(s t) \leqq \mu(f) \leqq \inf _{s} \sup _{t} f(s t) .
$$

Similarly, if $X$ is a right invariant subspace of $B(S)$ containing constants and $\mu$ is a right invariant mean on $X$, then for any $f \in X$,

$$
\sup _{s} \inf _{t} f(t s) \leqq \mu(f) \leqq \inf _{s} \sup _{t} f(t s) .
$$

We denote by $C(S)$ the Banach space of all bounded continuous real-valued functions on $S$ with supremum norm.

A continuous function $u: S \rightarrow C$ is said to be an almost-orbit of $\mathcal{S}=\left\{T_{t}\right.$ : $t \in S\}$ if

$$
\inf _{w} \sup _{t, s}\left\|u(s w t)-T_{s} u(w t)\right\|=0 .
$$

If an almost-orbit $u: S \rightarrow C$ of $\mathcal{S}=\left\{T_{t}: t \in S\right\}$ is bounded and $\mu$ is a mean on $C(S)$, then there exists a unique element $x_{\mu}$ of $H$ such that

$$
\mu_{t}(u(t), y)=\left(x_{\mu}, y\right) \quad \text { for all } y \in H .
$$

In fact, for each $y \in H$, 


$$
\left|\mu_{t}(u(t), y)\right| \leqq\|\mu\| \sup _{t}|(u(t), y)| \leqq \sup _{t}\|u(t)\|\|y\|
$$

and hence $\mu_{t}(u(t), \cdot)$ is a bounded linear functional on $H$. So, we obtain the desired result by the Riesz representation theorem.

Let $\left\{\mu_{\alpha}: \alpha \in A\right\}$ be a net of means on $C(S)$. Then $\left\{\mu_{\alpha}: \alpha \in A\right\}$ is said to be asymptotically invariant if for each $f \in C(S)$ and $s \in S$,

$$
\mu_{\alpha}(f)-\mu_{\alpha}\left(l_{s} f\right) \longrightarrow 0 \text { and } \mu_{\alpha}(f)-\mu_{\alpha}\left(r_{s} f\right) \longrightarrow 0
$$

THEOREM 1. Let $H$ be a real Hilbert space and let $C$ be a nonempty subset of $H$. Suppose that $S$ is a semitopological semigroup such that $C(S)$ has an invariant mean. Let $\mathcal{S}=\left\{T_{t}: t \in S\right\}$ be a nonexpansive semigroup on $C$. If an almost-orbit $u: S \rightarrow C$ of $\mathcal{S}=\left\{T_{t}: t \in S\right\}$ is bounded and $\bigcap_{s \in S} \overline{c o}\{u(s t): t \in S\} \subset C$, then the set $F(\mathcal{S})$ of all common fixed points of $T_{t}, t \in S$ is nonempty. Moreover, if $\left\{\mu_{\alpha}: \alpha \in A\right\}$ is an asymptotically invariant net of means on $C(S)$, then there exists an element $x_{0}$ of $F(S)$ such that $x_{\mu_{\alpha}}$ converges weakly to $x_{0}$, where $x_{\mu_{\alpha}}$ is an element of $H$ such that $\left(\mu_{\alpha}\right)_{t}(u(t), y)=\left(x_{\mu_{\alpha}}, y\right)$ for all $y \in H$.

Proof. Let $\mu$ be an invariant mean on $C(S)$. Then, there exists an element $x_{\mu}$ of $H$ such that $\mu_{t}(u(t), y)=\left(x_{\mu}, y\right)$ for all $y \in H$. We show $x_{\mu} \in$ $\bigcap_{s \in S} \overline{c o}\{u(s t): t \in S\}$. If not, we have $x_{\mu} \notin \overline{c o}\left\{u\left(s_{0} t\right): t \in S\right\}$ for some $s_{0} \in S$. By the separation theorem, there exists an element $y_{0}$ of $H$ such that

$$
\left(x_{\mu}, y_{0}\right)<\inf _{z \in \overline{c o}\left(u\left(s_{0} t\right): t \in S\right\}}\left(z, y_{0}\right) \text {. }
$$

So, we have

$$
\begin{aligned}
\left(x_{\mu}, y_{0}\right) & <\inf _{z \in \overline{c o}\left(u\left(s_{0} t\right): t \in S\right)}\left(z, y_{0}\right) \\
& \leqq \inf _{t \in S}\left(u\left(s_{0} t\right), y_{0}\right) \\
& \leqq \mu_{t}\left(u\left(s_{0} t\right), y_{0}\right) \\
& =\mu_{t}\left(u(t), y_{0}\right)=\left(x_{\mu}, y_{0}\right) .
\end{aligned}
$$

This is a contradiction. Therefore we have $x_{\mu} \in \bigcap_{s \in S} \overline{c o}\{u(s t): t \in S\}$, and hence $x_{\mu} \in C$.

Since $u$ is continuous and $\{u(t): t \in S\}$ is bounded, the real-valued function $t \rightarrow\|u(t)-y\|^{2}$ is in $C(S)$ for each $y \in H$. Let $r=\inf _{y \in H} \mu_{t}\|u(t)-y\|^{2}$ and $M=\{z \in H$ : $\left.\mu_{t}\|u(t)-z\|^{2}=r\right\}$. Since for each $y \in H$ and $t \in S$,

$$
\left\|x_{\mu}-y\right\|^{2}=\|u(t)-y\|^{2}-\left\|u(t)-x_{\mu}\right\|^{2}-2\left(u(t)-x_{\mu}, x_{\mu}-y\right),
$$

we have

$$
0 \leqq\left\|x_{\mu}-y\right\|^{2}
$$




$$
\begin{aligned}
& =\mu_{t}\|u(t)-y\|^{2}-\mu_{t}\left\|u(t)-x_{\mu}\right\|^{2}-2 \mu_{t}\left(u(t)-x_{\mu}, x_{\mu}-y\right) \\
& =\mu_{t}\|u(t)-y\|^{2}-\mu_{t}\left\|u(t)-x_{\mu}\right\|^{2} .
\end{aligned}
$$

Hence, for an element $y$ of $H$, it follows that $x_{\mu} \neq y$ if and only if $\mu_{t} \| u(t)-$ $x_{\mu}\left\|^{2}<\mu_{t}\right\| u(t)-y \|^{2}$. This implies that the set $M$ consists a single point $x_{\mu}$.

We prove that $x_{\mu} \in F(\mathcal{S})$. We first show that for every $s \in S$ and $y \in H$,

$$
\mu_{t}\|u(s t)-y\|^{2}=\mu_{t}\left\|T_{s} u(t)-y\right\|^{2} .
$$

Since $\{u(t): t \in S\}$ is bounded, there exists a positive number $M_{1}$ such that $\|u(t)\| \leqq M_{1}$ for any $t \in S$. Fix $t_{0} \in S$. Then we have

and hence

$$
\left\|T_{s} u(t)-T_{s} u\left(t_{0}\right)\right\| \leqq\left\|u(t)-u\left(t_{0}\right)\right\| \leqq 2 M_{1}
$$

$$
\left\|T_{s} u(t)\right\| \leqq\left\|T_{s} u\left(t_{0}\right)\right\|+2 M_{1} \quad \text { for all } t \in S .
$$

So, there exists a positive number $M_{2}$ such that $\left\|T_{s} u(t)\right\| \leqq M_{2}$ for all $t \in S$. Therefore, we have

$$
\begin{aligned}
\mid \mu_{t} \| & u(s t)-y\left\|^{2}-\mu_{t}\right\| T_{s} u(t)-y \|^{2} \mid \\
& =\left|\mu_{t}\left(\|u(s t)-y\|^{2}-\left\|T_{s} u(t)-y\right\|^{2}\right)\right| \\
& \leqq \mu_{t}\left(\left(\|u(s t)-y\|+\left\|T_{s} u(t)-y\right\|\right)\left|\|u(s t)-y\|-\left\|T_{s} u(t)-y\right\|\right|\right) \\
& \leqq\left(2\|y\|+M_{1}+M_{2}\right) \mu_{t}\left\|u(s t)-T_{s} u(t)\right\| \\
& \leqq\left(2\|y\|+M_{1}+M_{2}\right) \inf _{w} \sup _{t}\left\|u(s w t)-T_{s} u(w t)\right\|=0 .
\end{aligned}
$$

This implies $\mu_{t}\|u(s t)-y\|^{2}=\mu_{t}\left\|T_{s} u(t)-y\right\|^{2}$ for every $s \in S$ and $y \in H$. Using this, we have $x_{\mu} \in F(S)$. In fact,

$$
\begin{aligned}
\mu_{t}\left\|u(t)-T_{s} x_{\mu}\right\|^{2} & =\mu_{t}\left\|u(s t)-T_{s} x_{\mu}\right\|^{2} \\
& =\mu_{t}\left\|T_{s} u(t)-T_{s} x_{\mu}\right\|^{2} \\
& \leqq \mu_{t}\left\|u(t)-x_{\mu}\right\|^{2}=r,
\end{aligned}
$$

and hence $T_{s} x_{\mu} \in M$. Since $M=\left\{x_{\mu}\right\}$, we have $T_{s} x_{\mu}=x_{\mu}$ for all $s \in S$. Next, we prove that $x_{\mu}$ is independent of any invariant mean $\mu$ on $C(S)$. We know that $\mu_{t}\|u(t)-z\|^{2} \leqq \inf _{s} \sup _{t}\|u(t s)-z\|^{2}$ for all $z \in H$. On the other hand, fix $z \in F(\mathcal{S})$ and set $M_{3}=\sup \|u(t)-z\|$. Then, for any $\varepsilon>0$ there exists an $a$ in $S$ such that

$$
\sup _{s, t}\left\|u(t a s)-T_{t} u(a s)\right\|<\varepsilon \text {. }
$$

Since for each $s \in S$,

$$
\inf _{w} \sup _{t}\|u(t w)-z\|^{2} \leqq \sup _{t} \| u(\text { tas })-z \|^{2}
$$


we have

$$
\begin{aligned}
\leqq & \sup _{t}\left\|u(t a s)-T_{t} u(a s)\right\|^{2}+\sup _{t}\left\|T_{t} u(a s)-z\right\|^{2} \\
& +2 \sup _{t}\left\|u(t a s)-T_{t} u(a s)\right\|\left\|T_{t} u(a s)-z\right\| \\
\leqq & \varepsilon^{2}+\sup _{t}\left\|T_{t} u(a s)-T_{t} z\right\|^{2}+2 \varepsilon \sup _{t}\left\|T_{t} u(a s)-T_{t} z\right\| \\
\leqq & \varepsilon^{2}+\|u(a s)-z\|^{2}+2 \varepsilon M_{3},
\end{aligned}
$$

$$
\begin{aligned}
\inf _{w} \sup _{t}\|u(t w)-z\|^{2} \leqq \mu_{s}\|u(a s)-z\|^{2}+\varepsilon^{2}+2 \varepsilon M_{3} \\
\\
=\mu_{s}\|u(s)-z\|^{2}+\varepsilon^{2}+2 \varepsilon M_{3} .
\end{aligned}
$$

This implies $\inf _{w} \sup _{l}\|u(t w)-z\|^{2} \leqq \mu_{s}\|u(s)-z\|^{2}$ for all $z \in F(S)$. Then,

$$
\mu_{t}\|u(t)-z\|^{2}=\inf _{s} \sup _{t}\|u(t s)-z\|^{2} \quad \text { for all } z \in F(\mathcal{S}),
$$

and hence $x_{\mu}$ is independent of $\mu$. So, we denote the element $x_{\mu}$ by $x_{0}$.

Finally, for an asymptotically invariant net $\left\{\mu_{\alpha}: \alpha \in A\right\}$ of means on $C(S)$ we show that $w \lim x_{\mu_{\alpha}}=x_{0}$, where $x_{\mu_{\alpha}}$ is an element of $H$ such that $\left(\mu_{\alpha}\right)_{t}(u(t), y)=\left(x_{\mu_{\alpha}}, y\right)$ for all $y \in H$. Since $\left\|\mu_{\alpha}\right\|=1,\left\{\mu_{\alpha}: \alpha \in A\right\}$ has a cluster point $\mu$ in the sense of $w^{*}$-topology. Such a $\mu$ is an invariant mean. In fact, for each $\varepsilon>0, f \in C(S)$ and $s \in S$, there exists $\alpha_{0} \in A$ such that

$$
\left|\mu_{\alpha}(f)-\mu_{\alpha}\left(l_{s} f\right)\right| \leqq \frac{\varepsilon}{3} \quad \text { for all } \alpha \geqq \alpha_{0} .
$$

Since $\mu$ is a cluster point of the net $\left\{\mu_{\alpha}: \alpha \in A\right\}$, we can choose $\alpha_{1}\left(\geqq \alpha_{0}\right)$ such that

$$
\left|\mu_{\alpha_{1}}(f)-\mu(f)\right| \leqq \frac{\varepsilon}{3} \text { and }\left|\mu_{\alpha_{1}}\left(l_{s} f\right)-\mu\left(l_{s} f\right)\right| \leqq \frac{\varepsilon}{3}
$$

Hence, we have

$$
\begin{aligned}
&\left|\mu(f)-\mu\left(l_{s} f\right)\right| \leqq\left|\mu(f)-\mu_{\alpha_{1}}(f)\right|+\left|\mu_{\alpha_{1}}(f)-\mu_{\alpha_{1}}\left(l_{s} f\right)\right| \\
&+\left|\mu_{\alpha_{1}}\left(l_{s} f\right)-\mu\left(l_{s} f\right)\right| \\
& \leqq \\
& \frac{\varepsilon}{3}+\frac{\varepsilon}{3}+\frac{\varepsilon}{3}=\varepsilon .
\end{aligned}
$$

Since $\varepsilon>0$ is arbitrary, we have $\mu(f)=\mu\left(l_{s} f\right)$ for every $f \in C(S)$ and $s \in S$. This implies that $\mu$ is left invariant. Similarly, $\mu$ is right invariant. Since

$$
\begin{aligned}
\left\|x_{\mu_{\alpha}}\right\| & =\sup _{\|y\| \S 1}\left|\left(x_{\mu_{\alpha}}, y\right)\right|=\sup _{\|y\| \leq 1}\left|\left(\mu_{\alpha}\right)_{t}(u(t), y)\right| \\
& \leqq \sup _{t}\|u(t)\|,
\end{aligned}
$$

we get $\left\{x_{\mu_{\alpha}}: \alpha \in A\right\}$ is bounded by virtue of the boundedness of $\{u(t): t \in S\}$. 
Hence we can choose a subnet $\left\{x_{\mu_{\alpha} \beta}\right\}$ of the net $\left\{x_{\mu_{\alpha}}: \alpha \in A\right\}$ which converges weakly to some $z$ in $H$. If $\lambda$ is a cluster point of the net $\left\{\mu_{\alpha}\right\}$, then $\lambda$ is a cluster point of the net $\left\{\mu_{\alpha}\right\}$ and hence $\lambda$ is an invariant mean. Hence we obtain $z=x_{\lambda}=x_{0}$, which implies that $x_{\mu_{\alpha}}$ converges weakly to $x_{0} \in F(\mathcal{S})$.

Q.E.D.

3. Fixed point theorem. Let $X$ be a subspace of $B(S)$ containing constants. Then, according to Mizoguchi and Takahashi [5], a real-valued function $\mu$ on $X$ is called a submean on $X$ if it satisfies the following conditions:

(1) $\mu(f+g) \leqq \mu(f)+\mu(g)$ for every $f, g \in X$;

(2) $\mu(\alpha f)=\alpha \mu(f)$ for every $f$ and $\alpha \geqq 0$;

(3) for $f, g \in X, f \leqq g$ implies $\mu(f) \leqq \mu(g)$;

(4) $\mu(c)=c$ for every constant $c$.

For a submean $\mu$ on $X$, we also use $\mu_{t}(f(t))$ instead of $\mu(f)$.

LEMMA [5]. Let $S$ be a semitopological semigroup, let $X$ be a subspace of $B(S)$ containing constants and let $\mu$ be a submean on $X$. Let $\left\{x_{t}: t \in S\right\}$ be $a$ bounded subset of a Hilbert space $H$ and let $D$ be a closed convex subset of $H$. Suppose that for each $x \in D$, the real-valued function $f$ on $S$ defined by

$$
f(t)=\left\|x_{t}-x\right\|^{2} \quad \text { for all } t \in S
$$

belongs to $X$. Then, setting $g(x)=\mu_{t}\left\|x_{t}-x\right\|^{2}$ for all $x \in D$ and $r=\inf _{x \in D} g(x)$, there exists a unique element $z \in D$ such that $g(z)=r$ and $r+\|z-x\|^{2} \leqq g(x)$ for every $x \in D$.

Let $X$ be a subspace of $B(S)$ containing constants and invariant under $l_{s}, s$ $\in S$. Then a submean $\mu$ on $X$ is said to be left invariant if $\mu(f)=\mu\left(l_{s} f\right)$ for all $s \in S$ and $f \in X$.

TheOREM 2. Let $C$ be a nonempty subspace of a Hilbert space $H$ and let $S$ be a semitopological semigroup. Suppose that $X$ is a subspace of $B(S)$ containing constants and invariant under $l_{s}, s \in S$ and that there exists a left invariant submean $\mu$ on $X$. Let $\mathcal{S}=\left\{T_{t}: t \in S\right\}$ be a Lipschitzian semigroup on $C$ with Lipschitz constants $k_{s}, s \in S$, and let $u$ be an almost-orbit of $\mathcal{S}=\left\{T_{t}: t \in S\right\}$ such that $\{u(t): t \in S\}$ is bounded and $\bigcap_{s \in S} \overline{c o}\{u(s t): t \in S\} \subset C$. If for each $v \in H$, the real-valued function $f$ on $S$ defined by

$$
f(t)=\|u(t)-v\|^{2} \quad \text { for all } t \in S
$$

and the function $h$ on $S$ defined by

$$
h(t)=k_{t}{ }^{2} \quad \text { for all } t \in S
$$

belong to $X$ and $\mu_{t}\left(k_{t}{ }^{2}\right) \leqq 1$, then there exists an element $z \in C$ such that $T_{s} z=z$ for all $s \in S$. 
Proof. Define a real-valued function $g$ on $H$ by

$$
g(y)=\mu_{t}\|u(t)-y\|^{2} \quad \text { for all } y \in H .
$$

Then, setting $r=\inf _{y \in H} g(y)$, by Lemma, there exists a unique element $z \in H$ such that $g(z)=r$ and $r+\|z-y\|^{2} \leqq g(y)$ for every $y \in H$. For each $s \in S$, let $Q_{s}$ be the metric projection of $H$ onto $\overline{c o}\{u(s t): t \in S\}$. Then, by Phelps [6], $Q_{s}$ is nonexpansive and for each $t \in S$,

$$
\left\|u(s t)-Q_{s} z\right\|^{2}=\left\|Q_{s} u(s t)-Q_{s} z\right\|^{2} \leqq\|u(s t)-z\|^{2} .
$$

So, we have

$$
\begin{aligned}
\mu_{t}\left\|u(t)-Q_{s} z\right\|^{2} & =\mu_{t}\left\|u(s t)-Q_{s} z\right\|^{2} \leqq \mu_{t}\|u(s t)-z\|^{2} \\
& =\mu_{t}\|u(t)-z\|^{2},
\end{aligned}
$$

and thus $Q_{s} z=z$. This implies that $z \in \overline{c o}\{u(s t): t \in S\}$ for any $s \in S$, and hence $z \in \bigcap_{s \in S} \overline{c o}\{u(s t): t \in S\}$. We prove that $T_{s} z=z$ for all $s \in S$. Before proving it, we show $\mu_{t}\left\|u(s t)-T_{s} z\right\|^{2} \leqq k_{s}{ }^{2} \mu_{t}\|u(t)-z\|^{2}$ for every $s \in S$. Setting $k=\sup _{t \in S} k_{t}$ and $M=\sup _{t \in S}\|u(t)-z\|$, then for $\varepsilon>0$ there exists an $a \in S$ such that

$$
\sup _{t, s}\left\|u(s a t)-T_{s} u(a t)\right\|<\varepsilon .
$$

Since for each $t, s \in S$,

we obtain

$$
\begin{aligned}
\left\|u(s a t)-T_{s} z\right\|^{2} \leqq & \left\|u(s a t)-T_{s} u(a t)\right\|^{2}+\left\|T_{s} u(a t)-T_{s} z\right\|^{2} \\
& +2\left\|u(s a t)-T_{s} u(a t)\right\|\left\|T_{s} u(a t)-T_{s} z\right\| \\
\leqq & \varepsilon^{2}+k_{s}{ }^{2}\|u(a t)-z\|^{2}+2 \varepsilon k M,
\end{aligned}
$$

and hence

$$
\begin{aligned}
\mu_{t}\left\|u(s t)-T_{s} z\right\|^{2} & =\mu_{t}\left\|u(s a t)-T_{s} z\right\|^{2} \\
& \leqq \varepsilon^{2}+2 \varepsilon k M+k_{s}{ }^{2} \mu_{t}\|u(a t)-z\|^{2} \\
& =\varepsilon^{2}+2 \varepsilon k M+k_{s}{ }^{2} \mu_{t}\|u(t)-z\|^{2},
\end{aligned}
$$

$$
\mu_{t}\left\|u(s t)-T_{s} z\right\|^{2} \leqq k_{s}{ }^{2} \mu_{t}\|u(t)-z\|^{2} \quad \text { for all } s \in S .
$$

Since by Lemma

$$
\|z-y\|^{2} \leqq \mu_{t}\|u(t)-y\|^{2}-\mu_{t}\|u(t)-z\|^{2} \quad \text { for all } \quad y \in H,
$$

we have for each $s \subseteq S$

$$
\begin{aligned}
\left\|z-T_{s} z\right\|^{2} & \leqq \mu_{t}\left\|u(t)-T_{s} z\right\|^{2}-\mu_{t}\|u(t)-z\|^{2} \\
& =\mu_{t}\left\|u(s t)-T_{s} z\right\|^{2}-\mu_{t}\|u(t)-z\|^{2}
\end{aligned}
$$


and hence

$$
\leqq\left(k_{s}^{2}-1\right) \mu_{t}\|u(t)-z\|^{2},
$$

$$
\mu_{s}\left\|z-T_{s} z\right\|^{2} \leqq\left(\mu_{s}\left(k_{s}^{2}\right)-1\right) \mu_{t}\|u(t)-z\|^{2} \leqq 0 .
$$

This implies $\mu_{s}\left\|z-T_{s} z\right\|^{2}=0$. Since, for every $a, s \in S$,

we have

$$
\left\|z-T_{a} z\right\|^{2} \leqq 2\left\|z-T_{s} z\right\|^{2}+2\left\|T_{s} z-T_{a} z\right\|^{2},
$$

$$
\begin{aligned}
\left\|z-T_{a} z\right\|^{2} & \leqq 2 \mu_{s}\left\|z-T_{s} z\right\|^{2}+2 \mu_{s}\left\|T_{s} z-T_{a} z\right\|^{2} \\
& =2 \mu_{s}\left\|T_{a s} z-T_{a} z\right\|^{2} \\
& \leqq 2 k_{a}^{2} \mu_{s}\left\|T_{s} z-z\right\|^{2}=0 .
\end{aligned}
$$

This implies $T_{s} z=z$ for all $s \in S$.

Q.E.D.

\section{REFERENCES}

[1] S. Aizicovici, On the asymptotic behaviour of solutions of Voltera equations in Hilbert space, Nonlinear Analysis, 7 (1983), 271-278.

[2] H. Ishrhara, Fixed point theorems for lipschitzian semigroups, Canad. Math. Bull., 32 (1989), 90-97.

[3] A.T. LAU, Semigroup of nonexpansive mappings on a Hilbert space, J. Math. Anal. Appl., 105 (1985), 514-522.

[4] I. MiYadera and K. Kobayasi, On the asymptotic behavior of almost-orbits of nonlinear contraction semigroups in Banach spaces, Nonlinear Analysis, 6 (1982), 349-365.

[5] N. Mizoguchi and W. Takailashi, On the existence of fixed points and ergodic retractions for Lipschitzian semigroups in Hilbert spaces, Nonlinear Analysis, 14 (1990), 69-80.

[6] R.P. Phelps, Convex sets and nearest points, Proc. Amer. Math. Soc., 8 (1957), 790-797.

[7] G. RoDÉ, An ergodic theorem for semigroups of nonexpasive mappings in a Hilbert space, J. Math. Anal. Appl., 85 (1982), 172-178.

[8] W. TAKAHASHI, A nonlinear ergodic theorem for an amenable semigroup of nonexpansive mappings in a Hilbert space, Proc. Amer. Math. Soc., 81 (1981), 253-256.

[9] W. TAKAhashi, Fixed point theorems for families of nonexpansive mappings on unbounded sets, J. Math. Soc. Japan, 36 (1984), 543-553.

[10] W. TAKAhASHI, Fixed point theorem and nonliear ergodic theorem for nonexpansive semigroups without convexity, to appear in Canadian J. Math., 35(1992).

[11] W. TAKAhASH AND J.Y. PARK, On the asymptotic behavior of almost-orbits of commutative semigroups in Banach spaces, in "Nonlinear and Convex Analysis", pp. 271-293, Dekker, New York/Basel, 1987.

[12] W. TAKAHASHi AND P. J. ZHANG, Asymptotic behavior of almost-orbits of reversible semigroups of Lipschitzian mappings, J. Math. Anal. Appl., 142 (1989), 242-249.

Department of Information Science,

Tokyo Institute of Technology,

Oh-Okayama, Meguro-ku, Tokyo 152, Japan 